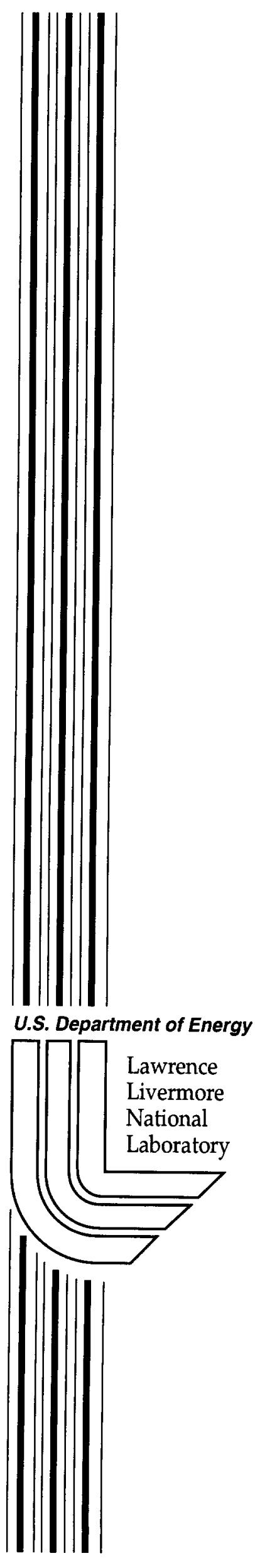

UCRL-ID-151370

\title{
New Approaches to Quantum Computing Using Nuclear Magnetic Resonance Spectroscopy
}

\author{
M. Colvin, V.V. Krishnan
}

February 7, 2003 


\section{DISCLAIMER}

This document was prepared as an account of work sponsored by an agency of the United States Government. Neither the United States Government nor the University of California nor any of their employees, makes any warranty, express or implied, or assumes any legal liability or responsibility for the accuracy, completeness, or usefulness of any information, apparatus, product, or process disclosed, or represents that its use would not infringe privately owned rights. Reference herein to any specific commercial product, process, or service by trade name, trademark, manufacturer, or otherwise, does not necessarily constitute or imply its endorsement, recommendation, or favoring by the United States Government or the University of California. The views and opinions of authors expressed herein do not necessarily state or reflect those of the United States Government or the University of California, and shall not be used for advertising or product endorsement purposes.

This work was performed under the auspices of the U. S. Department of Energy by the University of California, Lawrence Livermore National Laboratory under Contract No. W-7405-Eng-48.

This report has been reproduced directly from the best available copy.

Available electronically at http://www.doc.gov/bridge

Available for a processing fee to U.S. Department of Energy And its contractors in paper from

U.S. Department of Energy

Office of Scientific and Technical Information

P.O. Box 62

Oak Ridge, TN 37831-0062

Telephone: (865) 576-8401

Facsimile: (865) 576-5728

E-mail: reports@adonis.osti.gov

Available for the sale to the public from

U.S. Department of Commerce

National Technical Information Service

5285 Port Royal Road

Springfield, VA 22161

Telephone: (800) 553-6847

Facsimile: (703) 605-6900

E-mail: orders@ntis.fedworld.gov

Online ordering: http://www.ntis.gov/ordering.htm

OR

Lawrence Livermore National Laboratory

Technical Information Department's Digital Library

http://www.llnl.gov/tid/Library.html 


\title{
New Approaches to Quantum Computing using Nuclear Magnetic Resonance Spectroscopy.
}

\author{
Tracking Code: 00-LW-068 \\ Principal Investigator: Michael Colvin, BBRP \\ Co-Investigator: V. V. Krishnan, BBRP \\ Final Report: February 7, 2003 \\ UCRL-ID-
}

The power of a quantum computer $(\mathrm{QC})$ relies on the fundamental concept of the superposition in quantum mechanics and thus allowing an inherent large-scale parallelization of computation. In a QC, binary information embodied in a quantum system, such as spin degrees of freedom of a spin-1/2 particle forms the qubits (quantum mechanical bits), over which appropriate logical gates perform the computation. In classical computers, the basic unit of information is the bit, which can take a value of either 0 or 1 . Bits are connected together by logic gates to form logic circuits to implement complex logical operations. The expansion of modern computers has been driven by the developments of faster, smaller and cheaper logic gates. As the size of the logic gates become smaller toward the level of atomic dimensions, the performance of such a system is no longer considered classical but is rather governed by quantum mechanics. While a classical bit must be either 0 or 1 , a quantum bit or qubit simultaneously contains a 0 component and a 1 component. If the classical bits are denoted by $\mid 0>$ and $\mid 1>$, a quantum bit can be in any state $\alpha|0>+\beta| 1>$, where $\alpha$ and $\beta$ are complex number called amplitudes subject to the condition $|\alpha|^{2}+|\beta|^{2}=1$. In classical computers, a single string of $n$ zeros and ones would be needed to describe the state of $\mathbf{n}$ bits, while $\mathbf{n}$ quantum-mechanical bits can simultaneously represent $2^{n}$ numbers. Since qubits can evolve concurrently along many paths at once, which is known as the quantum parallelism, they could be used to perform many calculations on all the $2^{\mathrm{n}}$ bits at once. For example, in the case of two qubits, the states would be $\alpha|00>+\beta| 01>+\chi|10>+\delta| 11>$, with $\alpha, \beta, \chi$ and $\delta$ being complex amplitudes satisfying the condition $|\alpha|^{2}+|\beta|^{2}+|\chi|^{2}+|\delta|^{2}=1$. A consequence is that computations using quantum information can run exponentially faster than classical computers.

Quantum computers offer the potentially superior prospect of solving computational problems that are intractable to classical computers such as efficient database searches and cryptography. A variety of algorithms have been developed recently, most notably Shor's algorithm for factorizing long numbers into prime factors in polynomial time and Grover's quantum search algorithm. The algorithms that were of only theoretical interest as recently, until several methods were proposed to build an experimental QC. These methods include, trapped ions, cavity-QED, coupled quantum dots, Josephson junctions, spin resonance transistors, linear optics and nuclear magnetic resonance.

Nuclear magnetic resonance (NMR) is uniquely capable of constructing small QCs and several algorithms have been implemented successfully. NMR-QC differs from other implementations in one important way that it is not a single $Q C$, but a statistical ensemble of them. Thus, quantum computing based on NMR is considered as ensemble quantum computing. In NMR quantum computing, the spins with non-zero nuclear moments (spin $1 / 2$ nuclei such as ${ }^{1} \mathrm{H}$ or ${ }^{13} \mathrm{C}$ ) in an organic molecule dissolved in a solvent constitute the required qubits. The logic gates and algorithms correspond to set of instructions containing radio frequency (r.f) pulses and delays that manipulate the qubits and the final spectrum reflects the outcome of the algorithm. 
Three years ago, when we initiated proposal on NMR-QC, the foremost of the aim is to develop quantum computing as part of LLNL research programs and hence cultivate an interdisciplinary working group in the area of quantum computing. Our success in the proposal is in part responsible for the formation of the laboratory-wide exploratory group on 'quantum computing and information'. The PI's play an integral role in promoting the work performed using the LDRD funded project and hence acquire the attention within the lab as well outside.

In specific goals of the project were to (a) develop experimental and sample based methods to improve the performance of NMR-QC, (b) define and estimate actual time cost or efficiency of a QCs, and (c) construct a comprehensive simulator of QC based on the principles of ensemble quantum computing. We were able to accomplish these goals and in particular we have reached some significant milestones in defining the QC efficiency and development of the QC-simulator. These developments have resulted to three publications:

(1) Krishnan, V. V. (2001) Estimating the efficiency of ensemble quantum computing. Physics Letters $A$ 291, 27-33.

(2) George, J. A.; Colvin, M. E.; Krishnan, V. V. (2002) A simulator for ensemble quantum computing. Computer Physics Communications 144, 277-283.

(3) Murali, N and Krishnan, V.V. (2003) A Primer to nuclear magnetic relaxation in liquids. Concepts in Magnetic Resonance (in press).

The details of our accomplishments are discussed below.

\section{Improved sample conditions for NMR-QC.}

Since, the first independent reports by research groups from IBM and MIT on the feasibility of NMR spectroscopy in a two-qubit QC, several research groups around the world have contributed significantly to the growth of the field. One of the major efforts is to increase the total number of qubits, with the largest so far to seven. Systems with higher number of accessible qubits are often synthesized using special procedures. Isotopic enrichment of some of carbon $\left({ }^{13} \mathrm{C}\right)$ or nitrogen $\left({ }^{15} \mathrm{~N}\right)$ atoms selectively is a necessary step to increase the sensitivity of the NMR experiments. These compounds further dictate the need for special probes and spectrometer hardware. However, these systems must be considered as some of the major accomplishments in the field of quantum computing, their access to a standard NMR spectrometer is highly restricted. Thus, it was essential to develop experimental systems that can be used in a conventional NMR spectrometer environment.

The small molecules dissolved in a liquid crystal, orient either along or against the external magnetic field depending on the diamagnetic susceptibility of the medium. This partial alignment reintroduces the dipolar couplings between the spins that are averaged to zero in the isotropic solvent due to rotational Brownian motion. We have shown that these sample conditions provide an unique opportunity to increase the total number of accessible qubits in the NMR-QC. Summary of the results and the associated challenges are shown in Figure 1 . The simulated NMR spectrum of benzene in isotropic solvent is single line (Figure 1.a) and the same molecule shows multiple transitions in the liquid crystal medium (Figure 1.b). The spin system classification of benzene in the isotropic system is $A_{6}$, and becomes a AA'A" A" 'A" "A" ", spin system in the liquid crystal. Though this approach increases the total number of accessible spin states to perform quantum computing, the spectrum is too complex and the identity of a 'single qubit' is lost. This probably is the major bottleneck adopting such a system as a NMRQC, despite well-resolved resonances and strong dipolar couplings. During our work in progress, two independent works on the use liquid crystal medium NMR-QC in a two-qubit system have 
appeared. Demonstration of NMR-QC in the liquid crystal medium for more than two-qubits still remains to be significant challenge at large.

Figure 1. Simulated NMR spectrum of benzene. (a) Isotropic solvent, (b) liquid crystalline phase, and (c) selectively deuterium labeled benzene in liquid crystal solvent.

\section{Actual time cost (efficiency) of NMR-QC}

The computational performance of classical computers is often defined by number of floating point

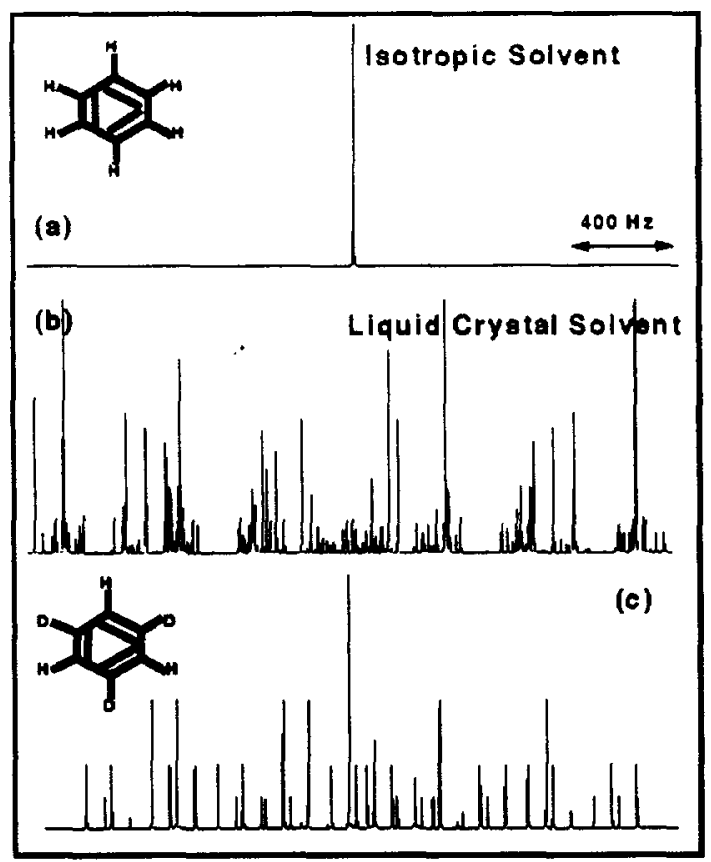
operations per second (flops). A similar definition for a quantum computer may not be meaningful, as the fundamental computing principles underlying conventional and quantum computers are different from each other. Thus, it may be necessary to define method to quantify the performance and efficiency of a QC. It is also important that such a definition must not only be restricted to NMR-QC and should easily be tailored for any quantum computer. The efficiency of ensemble quantum computing using magnetic resonance methods depends on two fundamental aspects: (a) the total length of the computational time that is purely determined by the decay of the superposition of states due to inherent thermal processes, and (b) how fast the spin states can be manipulated as defined by the interactions between the spins. The decay of the super-position of states is characterized by the spin-spin relaxation process defined by a characteristic relaxation time $T_{2}$, while the interaction between the spins is characterized by electron mediated indirect spin-spin coupling known as Jcoupling or direct dipolar coupling (D). Typically the $\mathrm{T}_{2}$ values are a maximum of a few seconds while the $J$ values range from $10-400 \mathrm{~Hz}$ and the dipolar couplings are an order of magnitude higher than the J-coupling values. The best systems (or molecular compounds) for ensemble quantum computing using NMR spectroscopy are the ones with long $T_{2}$ values and large coupling strengths. The focus of this aim was to develop methods to define and evaluate quantum-computing efficiency.

We have made an attempt to formulate a definition to determine the actual time cost of ensemble QC by defining a efficiency factor $(\mathrm{QC} \varepsilon)$. It is the first time such a definition has been introduced in this field and we have shown that it can be used to reliably accesses the performance and efficiency various quantum-computing methods. Our preliminary results demonstrate that quantum gates and algorithms that produce identical results differ significantly in their performance efficiency. As the NMR experiment progresses sequentially in time, the 
superposition of the coherent states available for computation is modulated by the set of operations performed on the various qubits. The product of various events (pulses, delays and pulsed field gradients), thus defines the total time of evolution of the spin system. Though, this quantifies the amount of NMR signal that is available at any given time during a particular information processing, in order to compare the performance of various quantum-computing algorithms this product must be normalized with the total experimental time. The final definition of $\mathrm{QC} \varepsilon$ is given by

$$
Q C_{\varepsilon}(t)=\frac{Q C_{c o h}(t)}{\sum_{i=1}^{N_{\tau}} \tau_{i}+\sum_{j=1}^{N_{\delta}} \delta_{j}+\sum_{k=1}^{N_{k}} \xi_{k}}=\frac{\prod_{i=1}^{N_{i}} e^{-\frac{\tau_{i}}{T_{2 \rho}}} \prod_{j=1}^{N_{\delta}} e^{-\frac{\delta_{j}}{T_{2 j}}} \prod_{k=1}^{N_{q}} e^{-\left(q_{k}^{2} D_{k}\right)}}{\sum_{i=1}^{N_{\tau}} \tau_{i}+\sum_{j=1}^{N_{\delta}} \delta_{j}+\sum_{k=1}^{N_{k}} \xi_{k}}
$$

The above equation describes the normalized decay of the qubit intensity for each of the pulse lengths $\left(\tau_{i}\right)$, delays $\left(\delta_{j}\right)$ and gradient lengths $\left(q_{k}\right)$, with their characteristic decay times given by relaxation times in the rotating frame $\left(T_{2 p j}\right)$, laboratory frame $\left(T_{2 j}\right)$ and the self diffusion constant $\left(D_{k}\right)$, respectively. $Q C_{\varepsilon}$ (units of inverse time, $s^{-1}$ ), quantifies the rate at which the quantum information processing is performed at any given moment of the processor and is also a measure of total amount of computation efficiency available at anytime during the processing.

This definition allows to monitor the performance of a $\mathrm{QC}$ as the code progresses using the advantages of generalized Bloch equations (numerically or analytically). Demonstration of the evaluation of the $\mathrm{QC}_{\varepsilon}$ in the performance of Grover's search algorithm for a three-qubit ensemble QC as function of the quantum computing code is shown in Figure 2. $\mathrm{QC}_{\varepsilon}$ is evaluated for the experimental system, ${ }^{1} \mathrm{H}^{13} \mathrm{C}^{19} \mathrm{FBr}$ (system a) on which the algorithm has been experimentally implemented is shown in continuous lines. For comparison purposes, the same evaluation performed on the backbone atoms of a peptide unit, $-{ }^{13} \mathrm{C}-{ }^{13} \mathrm{C}-{ }^{15} \mathrm{~N}$ (system $\mathrm{b}$ ) is shown in dashed lines. The three qubit Grover's search algorithm contains close to 3,500 of pulses and pulse-interrupted delays. The search algorithm is repeated several times and the end of each cycle is marked as either filled circles or squares in Figure 2. At the end of the first cycle of the Grover's search algorithm, the efficiency factor of the systems ' $a$ ' and ' $b$ ' are $0.949 \mathrm{~s}^{-1}$ and 0.741 $\mathrm{s}^{-1}$, respectively (inset of Figure 2), and at the end of the $8^{\text {th }}$ cycle the system ' $b$ ' have lost too much coherence and do not have enough sensitivity to continue the computation. Hence, the system ' $a$ ' is a better molecular system for $a$ three-qubit Grover's search algorithm.

\section{Figure 2. Plot of the efficiency of Grover's} search algorithm as implemented in the threequbit ensemble quantum computer using NMR spectroscopy as a function of the quantum computing code..

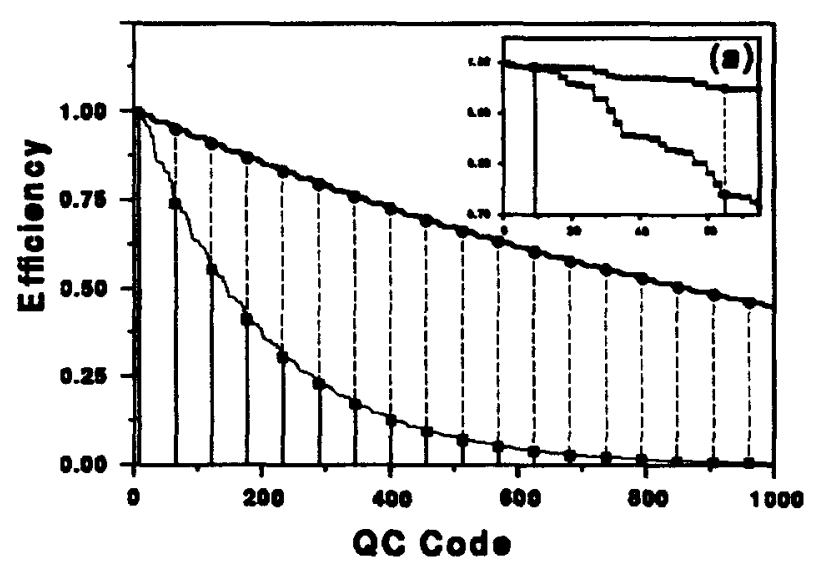

\section{Comprehensive QC simulator}

Simulation is an integral part of the design process of each new generation of microprocessors. Therefore, software to emulate the physical model representing the hardware implementation of a quantum information processor may prove essential. In conventional digital 
circuits, the internal architecture of each base unit is not directly relevant to the logical operations and algorithms, but dictates the speed and cost of the processor. In contrary, in a QC (independent of the physical implementation) every aspect of the system is critically involved in all the aspects of computation from how the qubit is defined to speed and cost. Therefore, it is essential to develop a physical model, by using the physics of the elementary units that constitute the QC. Theoretical work on quantum information processing usually assumes the existence of units that can perform ideal unitary operations, while the actual implementations of such operations are much more complicated. In particular, decoherence effects are often ignored. It is therefore, vital that theoretical tools to validate the physical designs are crucial towards construction of a practical QC.

The first version of the simulator (en-QClator) developed in our group demonstrates the feasibility of the approach. The principal flowchart of the comprehensive QC-simulator is shown in Figure 3. Each module was developed in a way such that additional improvements and modifications can be easily incorporated. Once the quantum algorithm and the related logical operations are identified the corresponding NMR-QC code, in terms of the pulse sequence can be designed.

Figure 3. Flow chart description of the proposed quantum computer simulator

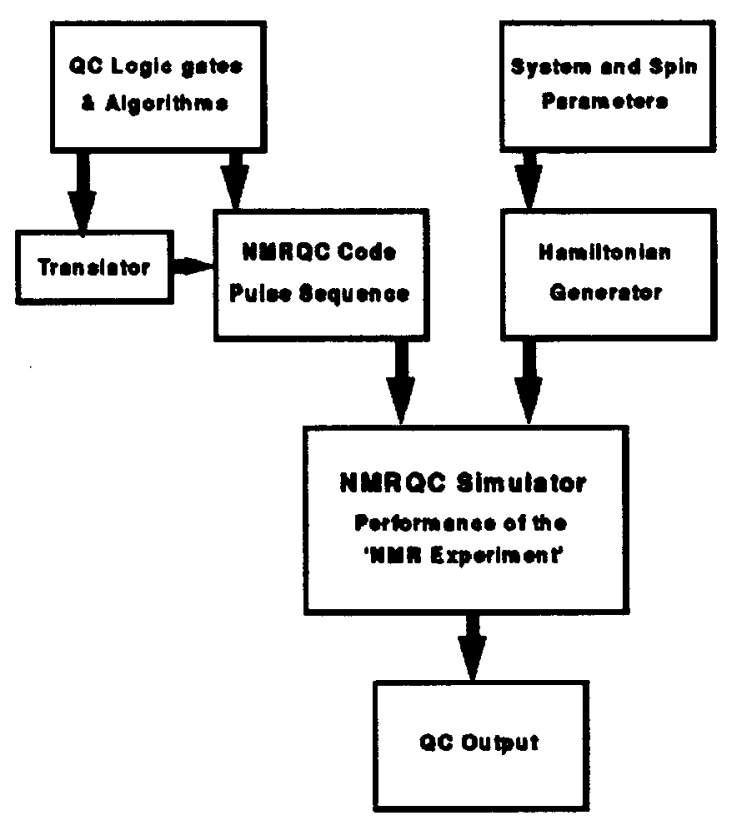

A translator that would convert QC-instruction sets to pulse-sequences performed this conversion. The spin and system parameters are then be used in the Hamiltonian generator module to construct the appropriate Hamiltonians for the NMR-QC code. Our initial version of the simulator uses of a numerical integration routine that evaluates the state of the spin systems at each time step as determined by the master equation in accordance to the QC code and system parameters. At the end of the execution of the QC code the state of the complete density matrix or only observable part of it can be piped to the output, which can be visualized either as a NMR spectrum or in the format a matrix format. The results of the QC-simulator demonstrating the CAT-state benchmark are shown in Figure 4.

The QC-simulator was primarily developed using $\mathrm{C}^{++}$(compiled using standard GNU) on a SGI $\mathrm{O}_{2}$ workstation, employing a MIPS R 10000 processor, within the IRIX environment. Perl 5 with CGI packages were also used to create a web-based interface for the simulation as well as to pipe the required output at the end of the simulation. 
Figure 4. Demonstration of the encoding and decoding parts of the Cat State benchmark evaluation of a three-qubit quantum computer using the enQC-lator. Arrow marks represent the direction of the flow of operations. Reference spectrum obtained after a selective $90^{\circ}$ pulse to $\mathrm{Q}_{1}$. Simulation for three different coupling topologies is also shown. The system parameters for this simulation are $v_{\mathrm{II}}=100$ $\mathrm{Hz}, v_{\mathrm{I} 2}=250 \mathrm{~Hz}, v_{\mathrm{I}}=425 \mathrm{~Hz}$ and $\mathrm{a}$ spectrometer operating frequency of 600 $\mathrm{MHz}$.

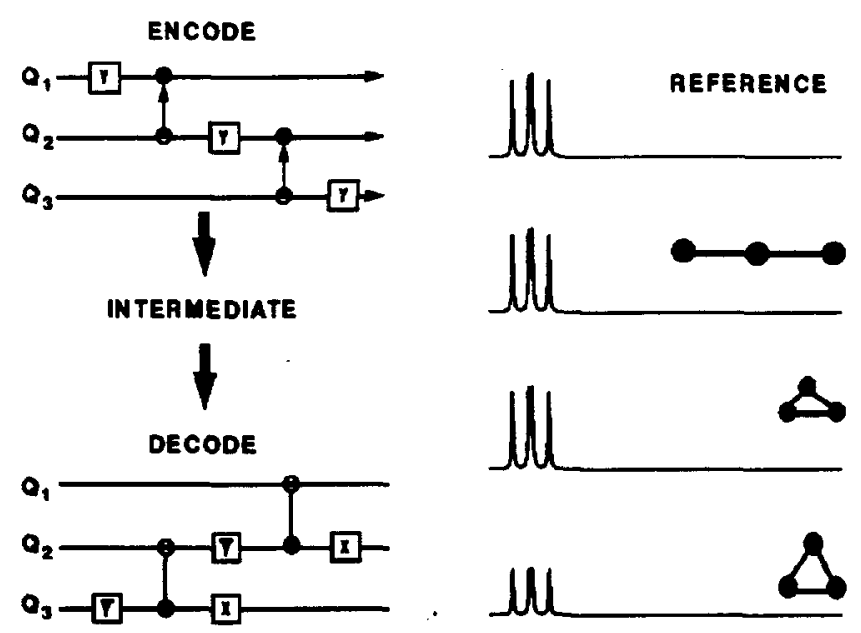

The QC-simulator, in addition to being a useful tool for our research purpose, it can serve a general purpose software to learn the fundamental principles of quantum computing itself. For example, researchers who are interested in developing quantum algorithms can directly use the QC-simulator to verify the practical aspects of implementation. It allows us to design, test and improve a quantum logical operation or algorithm and compare the theoretical derived protocols with experimental results. In addition, it can also be used to generate an experimental pulse sequences for a given quantum logic diagram either for simulation or for actual implementation in a NMR spectrometer. This code is available upon request from the PI's. We are also planning to release a public domain version of the program in the next few months.

\section{Summary}

The limiting factor of NMR-QC is the relaxation of the coherent states during the performance of the quantum computing. The principles of the relaxation of nuclear spins used in the NMR-QC are not straightforward. In the processes of developing the quantum computing efficiency, the collaboration with Varian resulted in a comprehensive introductory article in the relaxation of nuclear spins in the liquid state. In addition to the NMR, the next most promising experimental method for quantum computing is the use of 'quantum dot'. Solid-state NMR experiments helps to access the qubits formed by the quantum dots in the condensed matter. In order to explore the combined possibility of using solution and solid state NMR-QC, we are planning to resubmit a proposal to NSF in collaboration with Professor Risbud, Department of Chemical Engineering, UC Davis). These allowed us to have significant collaborations between LLNL and University as well as with Industry.

The LDRD allowed us the opportunity to carry out this exciting research for the past three years and initiate several fruitful collaborations with researchers in C\&MS, UC Davis, and Varian. This project had played a critical role in training several graduate and undergraduate students in an emerging field that interfaces the best of physics, computer science and engineering. We are planning to continue the work through inter-departmental collaborations at LLNL. In addition, we believe that this research will help lead to development of a comprehensive practical quantum computers of the future.

\section{Auspices}

This work was performed under the auspices of the U.S. Department of Energy by the University of California, Lawrence Livermore National Laboratory under Contract No. W-7405-Eng-48. 